\title{
Evaluación ecotoxicológica de sedimentos en una zona del Río Uruguay, con puntos finales indicadores de toxicidad aguda, sub-letal, crónica, reproductiva y teratogénica
}

\author{
Míguez, D. (1), Seoane, I. (1), Carrara, M. V. (1), Carnikián, A. (1), Keel, K. (1), Aizpún, A. (1), Bouvier, M. E. (2), Cartmell, E. ${ }^{(3)}$ \\ (1) Departamento Aguas y Productos Químicos. Laboratorio Tecnológico del Uruguay, LATU - (2) Departamento Aguas. \\ Unidad Fray Bentos. Laboratorio Tecnológico del Uruguay, Parque Industrial, Barrio Anglo, Fray Bentos, Río \\ Negro, Uruguay - ${ }^{(3)}$ School of Applied Sciences. Centre for Water Science, Cranfield University, Cranfield, Bedfordshire, \\ United Kingdom.
}

Contacto: dmiguez@latu.org.uy

Recibido: 12/07/2010 - Aprobado: 27/10/2010

\begin{abstract}
Resumen
El objetivo de este trabajo fue caracterizar la toxicidad de los sedimentos de una zona del Río Uruguay, aplicando una batería de bioensayos. Las muestras provinieron de: una zona de influencia de descargas domésticas y efluentes de una planta de celulosa (Fray Bentos), una ciudad turística aguas abajo (Las Cañas) y otra aguas arriba (Nuevo Berlín), sin influencia directa de dichos vertidos. Los bioensayos aplicados en elutriados fueron: de toxicidad con Photobacterium leiognathi, de reproducción con Ceriodaphnia dubia, de teratogenicidad y letalidad con Pimephales promelas y el ensayo sub-letal en sedimento completo con Hyalella curvispina. Para evaluar la biodisponibilidad y las rutas de toxicidad, se midió el fraccionamiento del 2,4,5-triclorofenol, con resultados: $63 \%$ disuelto, $27 \%$ adsorbido sobre partículas y el resto sedimentado. Se observó toxicidad por orgánicos y metales con P. leioghnati para todos los sitios. La frecuencia de malformaciones vertebrales de P. promelas fue de 3.3\% en Fray Bentos, pero C. dubia sólo mostró efectos en Nuevo Berlín y Las Cañas. Los efectos con H. curvispina se evidenciaron en todas las muestras. Las rutas de toxicidad incluirían la ingestión de contaminantes disueltos y particulados asociados a arcillas y limo. El vínculo causa-efecto para la toxicidad se continuará investigando. Palabras clave: Bioensayos, elutriado, teratogenicidad, clorofenoles.
\end{abstract}

\begin{abstract}
$\underline{\text { Abstract }}$
The aim of this work was to characterize the sediment toxicity of a zone of the River Uruguay through a battery of bioassays. The samples were extracted from an area of influence of domestic wastewater discharges and pulp mill effluents (Fray Bentos), a touristic town downstream (Las Cañas), and another one, upstream, isolated from those discharges (Nuevo Berlín). The bioassays: Photobacterium leiognathi toxicity screen, Ceriodaphnia dubia reproduction and embryo-larval teratogenicity and lethality with Pimephales promelas were applied to the sediment elutriates, while the Hyalella curvispina sub-lethal growth test was performed using whole sediment. To assess bioavailability and toxicity pathways, the sediment-water partitioning of 2,4,5-triclorofenol among phases was determined as: $63 \%$ dissolved, $27 \%$ suspended and the rest settled. Toxicity to organics and metals using $P$. leioghnati was observed in all sampled sites. The frequency of $P$. promelas vertebral malformations was 3.3\% only to Fray Bentos elutriate, but the C. dubia reproduction bioassay showed effects only in those from Nuevo Berlín and Las Cañas. The growth test with $H$. curvispina evidenced effects for all samples. The toxicity pathways could be ingestion of dissolved and particulate contaminants, possibly associated to higher clay and silt contents and contaminant input. The cause-effect relationships will be subject to further research.

Keywords: Bioassays, elutriate, teratogenicity, chlorophenols.
\end{abstract}

\section{Introducción}

El Río Uruguay es uno de los más grandes ríos de América del Sur, sustento de una rica biodiversidad acuática. Su importancia para el ser humano radica además en los usos domésticos, industriales, agrícolas, pesqueros y turísticos de este recurso hídrico de importancia binacional. Por consiguiente, es de crucial importancia entender si alguna descarga puntual al río, como podrían ser las provenientes de los efluentes de una planta de celulosa blanqueada, las aguas residuales cloacales de las ciudades asentadas a sus márgenes o las provenientes de fuentes no-puntuales debido al uso de agroquímicos usados en plantaciones de soja, podrían afectar la calidad del agua.

La biodisponibilidad de los compuestos químicos es un aspecto que debería ser tenido más en cuenta en las evaluaciones ecotoxicológicas y de riesgos (Martín-Díaz et al., 2006). Por este motivo, el estudio de las características fisicoquímicas de las fracciones agua y sedimento en el Río Uruguay es necesaria para evaluar la biodisponiblidad y el fraccionamiento, para así poder estimar mejor el potencial de ecotoxicidad de los contaminantes vertidos en los efluentes industriales y domésticos y, por tanto, la calidad del agua del río. Como las propiedades fisicoquímicas pueden variar entre sedimentos de 
diferentes sitios, afectando la partición y por ende la biodisponibilidad y ecotoxicidad de los posibles contaminantes (Kendal et al., 2001, p. 1016), es pertinente caracterizar específicamente las propiedades de los sedimentos y la exposición a los contaminantes en varios sitios del Río Uruguay, incluyendo aquellas áreas potencialmente afectadas por actividades industriales y las descargas de aguas residuales cloacales, en comparación con áreas de referencia que no están recibiendo estas descargas puntuales.

Primeramente se debería considerar la ruta de ingreso de los contaminantes al organismo. Esta puede ser directamente por pasaje de las sustancias tóxicas disueltas a través de las membranas, ruta principal de exposición para la mayoría de los organismos bentónicos, pero también puede ser mediante la ingestión de partículas de sedimento contaminadas (NAS, 2003). Los contaminantes hidrofóbicos adsorbidos a partículas o al carbono disuelto pueden desorberse, ocasionando así la re-exposición de los organismos. Finalmente, la bioacumulación de contaminantes en los tejidos de la biota, puede conducir a biomagnificación a través de la cadena trófica (Katagi, 2006; USEPA, 2000)

Para predecir la bioacumulación de una sustancia se debe considerar la distribución entre las fracciones disueltas y suspendidas (Voutsas et al., 2001). El coeficiente de partición octanol-agua (log $\mathrm{K}_{\mathrm{ow}}$ ) es usado como indicador de potencial de acumulación, y la bioacumulación es inversamente proporcional a este coeficiente. Las sustancias lipofílicas se adsorben a las partículas de sedimento (Katagi, 2006). En general, un compuesto tiende a distribuirse en la materia orgánica si $\log \mathrm{K}_{\mathrm{ow}}>1$. En el caso de los clorofenoles el $\log \mathrm{K}_{\mathrm{ow}}>2$, por lo que se distribuirían en la fase sedimento. Los clorofenoles pueden contaminar el agua, los suelos y los sedimentos. Algunos se los puede hallar en los efluentes de pulpa de celulosa blanqueada (ATSDR, 1998; Ohlenbusch et al., 2000). Los halogenuros orgánicos extraíbles (EOX) están formados por esta clase de compuestos y otras sustancias que puedan ser extraídas de las fases sólidas con acetato de etilo, en tanto los halogenuros orgánicos adsorbibles (AOX) representan a los compuestos orgánicos halogenados que se pueden extraer del agua mediante carbón activado. El compuesto 2,4,5-triclorofenol (2,4,5TCP) fue elegido en nuestra investigación como sustancia modelo para estimar el fraccionamiento de los compuestos orgánicos entre el agua y el sedimento a través de la relación AOX/EOX. Esta relación ha sido utilizada por varios autores para evaluar los impactos de efluentes de pulpa de celulosa y las características espacio-temporales de la contaminación de agua y sedimentos en la cercanía de las descargas industriales (Saski et al., 1997; Sibley et al., 1998).

Las concentraciones de contaminantes en los distintos compartimientos ambientales pueden predecirse utilizando bases de datos de propiedades fisicoquímicas y características de toxicidad, y/o realizando experimentos de exposición en el medio acuático. El uso de una batería de bioensayos a varios niveles tróficos se recomienda por incluir diferentes mecanismos de toxicidad y sensibilidades para evaluar los riesgos ambientales específicos para los sitios (Chapman, 2002). Por ejemplo, las bacterias son más sensibles a los metales que los peces y los seres humanos (UNESCO, WHO, UNEP, 1996). Algunos contaminantes químicos en los organismos no pueden ser determinados cuantitativamente en los tejidos por su rápida metabolización; es por eso que el estudio de efectos de toxicidad subletal en los organismos provee importante información en estos casos. Los efectos tóxicos de mezclas de contaminantes a los que la biota está expuesta en el ambiente podrían no ser predecibles empleando únicamente datos de concentraciones tisulares (Lower y Kendall, 1990, citados por Kendal et al., 2001). Las fases embrio-larvales en organismos acuáticos son las más sensibles en su ciclo de vida
(Weis y Weis, 1987). Las alteraciones de las funciones homeostásicoendócrinas en el desarrollo temprano pueden estar influenciadas por muchos procesos fisiológicos durante la vida de un organismo (Warner y Jenkins, 2007), de modo que los bioensayos con peces representan una muy promisoria alternativa para el ensayo de toxicidad acuática (Halder et al., 2010).

Este trabajo evalúa la ecotoxicidad de los sedimentos de una zona del Río Uruguay y examina la influencia de las características físicas y químicas de los mismos en el fraccionamiento y biodisponibilidad de los compuestos orgánicos clorados. El diseño experimental utilizado fue creado para servir como herramienta de evaluación de la exposición de organismos acuáticos a contaminantes ambientales, para posteriormente evaluar los riesgos en forma más exacta que por medio de bases de datos, ya que en forma experimental se reflejan las condiciones de los sitios.

\section{Materiales y Métodos}

El diseño experimental se compuso por métodos ecotoxicológicos, químicos y físicos (Figura 1). Se analizaron los nutrientes (N, P, materia orgánica), los compuestos orgánicos halogenados extraíbles (EOX) y tamaño de partícula en sedimentos. Se realizó un elutriado y se analizó compuestos orgánicos halogenados adsorbibles (AOX) en dicha fracción. El bioensayo con Hyalella curvispina fue aplicado al sedimento completo mientras que a la fracción acuosa se le aplicó una batería de bioensayos compuesta por Photobacterium leiognathi, Ceriodaphnia dubia y Pimephales promelas.

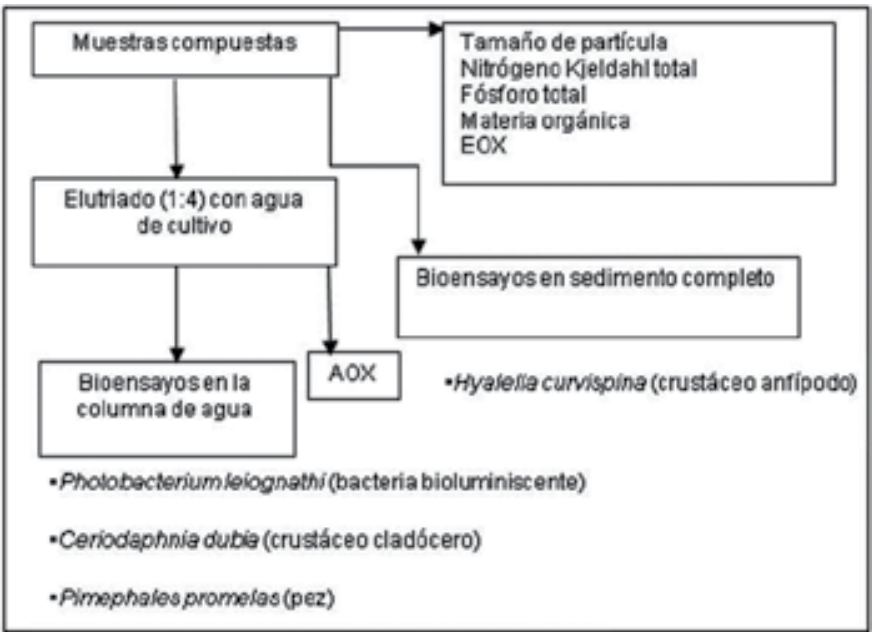

Figura 1. Diseño experimental para la evaluación del fraccionamiento de sustancias organocloradas y la toxicidad por exposición de organismos acuáticos a sedimentos y elutriados extraídos de sitios del Río Uruguay cercanos a las localidades de Nuevo Berlín, Fray Bentos y Las Cañas, departamento de Río Negro, Uruguay.

\section{Muestreo y preparación del elutriado}

Las muestras de sedimentos del fondo fueron extraídas con draga Petit Ponnar en transectas del río en tres zonas: Nuevo Berlín (sitio control), Fray Bentos (sitio cercano a la ciudad e inmediatamente aguas abajo de la planta de celulosa) y Las Cañas (ciudad turística, aguas debajo de Fray Bentos). Las porciones de las muestras de cada transecta central fueron mezcladas para preparar muestras compuestas representativas de cada área de muestreo. Para realizar el elutriado se colocó una porción apropiada de cada muestra de sedimento $(1.5 \mathrm{~kg}$ aproximadamente) en un recipiente de acero inoxidable de 10 litros y 
se mezcló en forma mecánica con 6 litros de agua de dilución durante 30 minutos utilizando un agitador de paleta (Janke y Kunkel modelo RW20, IKA-WERK, Staufen, Germany) (US EPA, 1998).

El agua de dilución se obtuvo por filtración de agua potable a través de un sistema de filtros compuesto por un filtro mecánico de 5 $\mu \mathrm{m}$, carbón activado de $20 \mu \mathrm{m}$ y $5 \mu \mathrm{m}$ y luz UV (TGI, TGI-525, BreaCalifornia, Estados Unidos), con las siguientes características: dureza total $\left(\mathrm{mg} \mathrm{CaCO}_{3} / \mathrm{l}\right)>25$, oxígeno disuelto $(\mathrm{mg} / \mathrm{l})>60 \%$, $\mathrm{pH} 7-8$.

\section{Estudio del fraccionamiento de AOX/EOX}

La muestra de sedimento correspondiente a Las Cañas fue adicionada con una solución de 2,4,5-triclorofenol (preparada pesando $0.3827 \mathrm{~g}$ de 2,4,5-triclorofenol en $200 \mathrm{ml}$ de metanol). Una submuestra de $1.5 \mathrm{~kg}$ del sedimento compuesto fue mezclada con 6 litros de agua de dilución y $50.0 \mathrm{ml}$ de la solución de 2,4,5-triclorofenol. Se analizó el tamaño de las partículas en suspensión por difracción láser. El AOX disuelto fue analizado después de filtrar el elutriado a través de filtro de microfibra de vidrio de porosidad $0.45 \mu \mathrm{m}$. El EOX se analizó en la materia depositada en los filtros y se tomaron 54 y $125 \mathrm{ml}$ para su análisis.

\section{Ensayos físicos}

Los tamaños de partícula fueron medidos por el método de difracción láser (UOP, 2007) usando un equipo trilaser Microtrac modelo S3500 (Microtrac Inc., Florida, Estados Unidos).

\section{Análisis químicos}

Los EOX de los sedimentos fueron analizados extrayendo por sonicado aproximadamente $1 \mathrm{~g}$ de la muestra húmeda con acetato de etilo, pureza $>99.5 \%$ (Mallinckrodt Chemicals, Xalostoc, México) (USEPA, 1996). Una alícuota de $25 \mu \mathrm{l}$ del extracto fue directamente colocada en el horno de pirólisis, usando una corriente de $\mathrm{O}_{2} / \mathrm{Ar}(150: 200 \mathrm{ml} / \mathrm{min})$ y el halogenuro de hidrógeno (HX) fue determinado por titulación microcoulométrica con un equipo de combustión/coulombimetría (Mitsubishi TOX 100, COSA Instrument, Norwood, Estados Unidos). Los AOX de las muestras acuosas fueron determinados por el método de combustión/coulombimetría (ISO 9562:2004) después de la adsorción de la solución, a través de un sistema de pasaje de flujo compuesto por columnas de carbón activado. El estándar de calibración de AOX y EOX fue 2,4,5-triclorofenol 98\% puro (Aldrich, Francia). El fósforo total fue analizado por el método de digestión sulfonítrica manual, seguida por el método de inyección en flujo (FIA) (Míguez et al., 2009), empleando el equipo QuikChem 8500 (Lachat Instruments, Loveland, Estados Unidos). El límite de detección (LD) fue $6 \mathrm{mg} / \mathrm{kg}$.

El nitrógeno Kjeldahl total fue determinado digiriendo $1 \mathrm{~g}$ de la muestra de sedimento secada al aire por el método clásico basado en la norma ISO 5663:1984, adaptado a tubos de Kjeldhal semi-micro usando un digestor en bloque de digestión (Gerhardt; GmbH \& Co. $\mathrm{KG}$; Königswinter, Alemania) trabajando a $410{ }^{\circ} \mathrm{C}$. La solución fue entonces titulada con $\mathrm{HCl} 0.01 \mathrm{~N}$ con indicador mixto compuesto por verde de bromocresol y rojo de metilo, virando desde el verdoso al violeta grisáceo. $\mathrm{LD}=0.7 \mathrm{mg} / \mathrm{kg}$.

El contenido de material orgánico de los sedimentos fue medido en mufla, a $400{ }^{\circ} \mathrm{C}$ (Burt, 2004).

\section{Bioensayos}

Los tests ecotoxicológicos in vivo e in vitro aplicados a los elutriados del sedimento fueron realizados en las condiciones resumidas en la Tabla 1.

La clasificación de las anormalidades en embriones y larvas de $P$. promelas fue de acuerdo a Lugowska (2007) y Jezierska et al. (2009) para embriones de Cypriniformes. Se obtuvo fotografías de las larvas normales de P. promelas usando lupa binocular Olympus SZ61 y de las larvas anómalas con microscopio óptico Leitz y software Motic images pro Plus 2.0 ML.

\begin{tabular}{|c|c|c|c|c|}
\hline $\begin{array}{c}\text { Nombre } \\
\text { de la especie }\end{array}$ & $\begin{array}{l}\text { Phosphobacterium } \\
\text { leioghnati }\end{array}$ & $\begin{array}{l}\text { Hyalella } \\
\text { curvispina }\end{array}$ & $\begin{array}{l}\text { Ceriodaphnia } \\
\text { dubia }\end{array}$ & $\begin{array}{l}\text { Pimephales } \\
\text { promelas }\end{array}$ \\
\hline Estadio & Cultivo bacteriano & Juvenil (7-14 días) & Neonatos $(<24 \mathrm{~h})$ & Embriones \\
\hline $\mathrm{N}^{0}$ organismos & Cultivo bacteriano & 10 por acuario & $\begin{array}{l}1 \text { neonato por vaso } \\
10 \text { por dilución }\end{array}$ & 10 por acuario \\
\hline Volumen de muestra & $5 \mathrm{ml}$ & $100 \mathrm{ml}$ & $15 \mathrm{ml}$ & $70 \mathrm{ml}$ \\
\hline Tipo de muestra & Elutriado & $\begin{array}{l}\text { Sedimento completo } \\
\text { Control con agua de dilución } \\
\text { y malla de } 625 \mu \mathrm{m}\end{array}$ & Elutriado & Elutriado \\
\hline Régimen & Estático & Estático & $\begin{array}{c}\text { Renovación } \\
\text { diario, semiestático }\end{array}$ & $\begin{array}{c}\text { Renovación } \\
\text { diario, semiestático }\end{array}$ \\
\hline $\mathrm{N}^{0}$ réplicas & 2 & 4 & 10 & 4 \\
\hline Diluciones (\%) & $80,40,20,10,5,2.5$ & Logarítrmica & Geométrica (a partir de 50\%) & Geométrica \\
\hline Punto final & Medida de la luminiscencia & Sobrevivencia y crecimiento & Sobrevivencia y crecimiento & Teratogenicidad; letalidad \\
\hline Tiempo de exposición & $30 \mathrm{~min}$ & 10 días & Hasta 3 puestas (7-8 días) & 7 días \\
\hline Temperatura $\left({ }^{\circ} \mathrm{C}\right)$ & $30 \pm 2$ & $23 \pm 2$ & $23 \pm 2$ & $20 \pm 2$ \\
\hline $\begin{array}{c}\text { Fotoperíodo } \\
\text { (luz: oscuridad, horas) }\end{array}$ & No & $16: 8$ & $16: 8$ & $16: 8$ \\
\hline Aireación & No & Sí & No & No \\
\hline Referencias & Manual PCB-Checklight & $\begin{array}{c}\text { US EPA 600/R-99/064 } \\
\text { Test Method 100.1, 2000, } \\
\text { modificado }\end{array}$ & $\begin{array}{c}\text { US EPA 821-R-02-013 } \\
\text { Test Method 1002.0, 2002, } \\
\text { modificado }\end{array}$ & $\begin{array}{l}\text { US EPA 821-R-02-013 } \\
\text { Test Method 1001.0 }\end{array}$ \\
\hline
\end{tabular}

Tabla 1. Determinación del \% de humedad. 


\section{Resultados}

\section{Ensayos físicos y químicos}

Como se ve en la Figura 2, las muestras de Nuevo Berlín y de Fray Bentos tuvieron mayor contenido de arcilla y de limo que la de Las Cañas $(55.2,51.5,27.8 \mathrm{~g} / 100 \mathrm{~g}$, respectivamente).
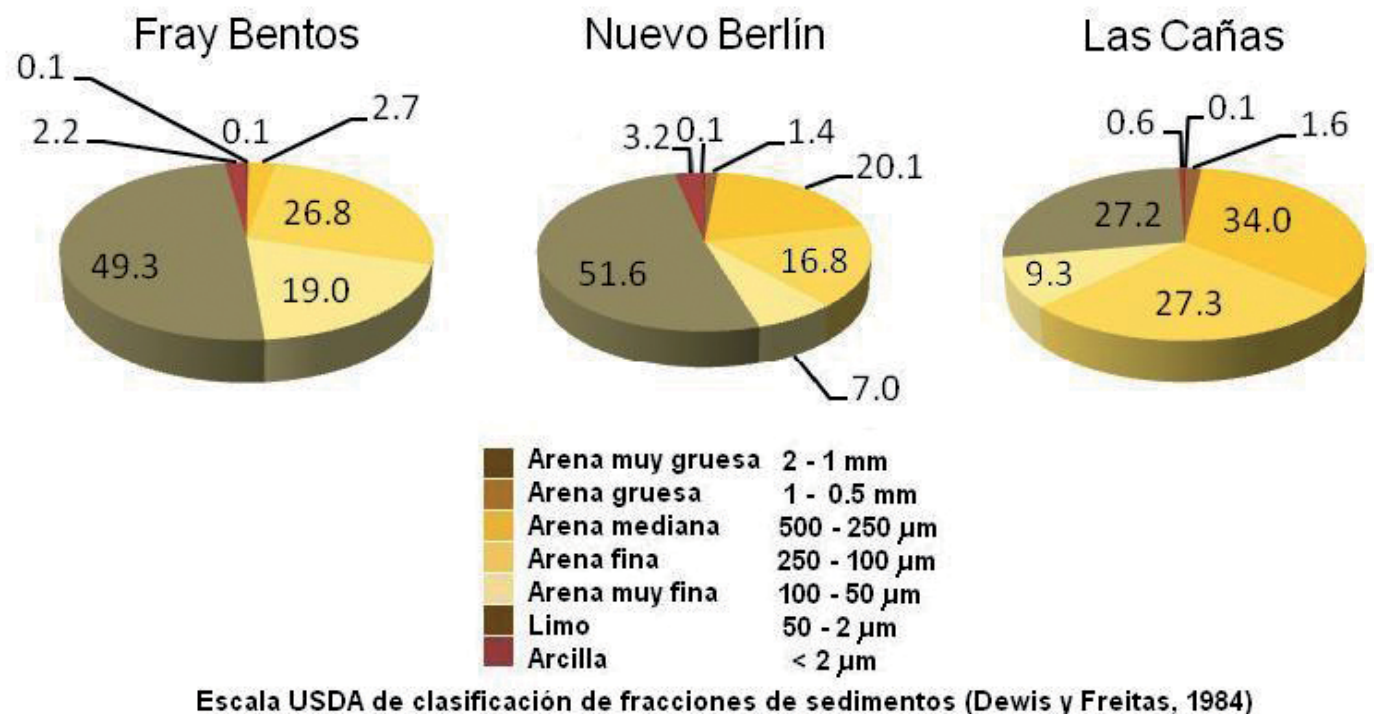

Figura 2. Distribución granulométrica de los sedimentos de la transecta central en tres sitios del Río Uruguay.

Las concentraciones de los nutrientes se expresan en la Tabla 2. La materia orgánica tiene una concentración similar en todos los sitios, pero el fósforo y el nitrógeno son más altos en la muestra de Fray Bentos ( $2164 \mathrm{mg} / \mathrm{kg}$ comparado con $144 \mathrm{mg} / \mathrm{kg}$ del control y 290 frente a $151 \mathrm{mg} /$ $\mathrm{kg}$, respectivamente). EOX y AOX fueron no detectables en todos los sitios en las muestras sin enriquecer.

\begin{tabular}{|c|c|c|c|c|c|}
\hline Código de muestra & $\begin{array}{c}\text { Fósforo total } \\
(\mathbf{m g} / \mathbf{k g})(\mathbf{c o m o})\end{array}$ & $\begin{array}{c}\text { Nitrógeno Kjeldahl total } \\
(\mathbf{m g} / \mathbf{k g})(\mathbf{c o m o})\end{array}$ & $\begin{array}{c}\text { Materia orgánica } \\
\text { (g/100 g) }\end{array}$ & $\begin{array}{c}\text { EOX } \\
\text { (como Cl) (mg/kg) } \\
\text { (LD: 20ug/kg) }\end{array}$ & $\begin{array}{c}\text { AOX } \\
\text { (como Cl) (mg/l) } \\
\text { (fase acuosa) } \\
(\mathbf{L D} \text { : 7ug/L) }\end{array}$ \\
\hline Nuevo Berlín & 144 & 151 & 1,1 & ND \\
\hline Fray Bentos & 2164 & 290 & 1,0 & ND & ND \\
\hline Las Cañas & 457 & 116 & 0,6 & ND & ND \\
\hline
\end{tabular}

ND: No detectable - LD: límite de detección

Tabla 2. Análisis químico de las muestras extraídas por draga en tres sitios del Río Uruguay

\section{Resultados del estudio de fraccionamiento}

La concentración adicionada teórica de AOX fue $8.58 \mathrm{mg} / 1$, como Cl. En el elutriado quedó $5.37 \mathrm{mg} / \mathrm{l} \mathrm{AOX}(63 \%)$ y el EOX fraccionado entre las partículas $(2.31 \mathrm{mg} / \mathrm{l} ; 27 \%)$ y el resto en las partículas sedimentadas. Al separarse el elutriado y el sedimento se pudo observar que una parte importante de las partículas más finas (arcilla y limo) permanecieron suspendidas en el líquido y los tamaños de las partículas en suspensión medidos por difracción láser fueron del rango de 1 a $7 \mu \mathrm{m}$ en un $99 \%$. 


\section{Resultados de los bioensayos}

El screening con P. leighonati tuvo los siguientes resultados de CI50 a los 30 minutos para toxicidad por metales y compuestos orgánicos, respectivamente: Nuevo Berlín: 19 y 37\%: Fray Bentos: 18 y 2\% y Las Cañas: 35 y 30\%. Se considera que la concentración de inhibición 50\% (CI50) es el umbral de toxicidad (manual PCB Checklight).

En el bioensayo con H. curvispina en sedimento completo se encontraron diferencias significativas en el peso seco por individuo promedio de las tres muestras analizadas respecto al control (ANOVA, $\mathrm{p}=0,023,95 \%$ confianza). Se evaluó la diferencia entre las medias de cada par de datos mediante la prueba de múltiples rangos y se hallaron diferencias significativas entre cada una de las tres áreas contra el control, usando el estadístico de prueba de diferencia mínima significativa (DMS) de Fisher al 5\% de significancia (Steel y Torrie, 1993).

Los resultados para el ensayo de reproducción con C. dubia de tres generaciones mostraron que habría efectos crónicos, ya que la concentración de inhibición 25\% (CI25\%) en Nuevo Berlín fue de 20.3\% y en Las Cañas 27.0\%, mientras que en Fray Bentos fue >50\% (no tóxico en forma crónica para el punto final de reproducción). Software ICPin (USEPA) y ToxCalc.

\begin{tabular}{|c|c|c|c|}
\hline & \multicolumn{2}{|c|}{ Toxicidad crónica } & \multirow{2}{*}{$\begin{array}{c}\begin{array}{c}\text { Toxicidad } \\
\text { aguda }\end{array} \\
\text { CL50 (48 h) }\end{array}$} \\
\hline & CI25 (\%) & $\begin{array}{l}\text { CL (punto final de } \\
\text { tres generaciones) }\end{array}$ & \\
\hline Nuevo Berlín & $\begin{array}{c}20,3 \\
C I(95)=12,5-25,0\end{array}$ & $>50$ & $>50$ \\
\hline Fray Bentos & $>50$ & $>50$ & $>50$ \\
\hline Las Cañas & $\begin{array}{c}27,0 \\
C I(95)=18,4-38,0\end{array}$ & $\begin{array}{c}40,3 \\
\text { CI }(95)=29,7-54,8\end{array}$ & $>50$ \\
\hline
\end{tabular}

CL: concentración letal - Cl: concentración de inhibición

Tabla 3. Resultados con el bioensayo con $C$. dubia de tres generaciones

\section{Ensayo con P. promelas con embriones y larvas}

La mortalidad para Nuevo Berlín fue 21.5\% y en Fray Bentos, 7.5\%. En Las Cañas no hubo mortalidad. En Nuevo Berlín y en Las Cañas no se encontraron individuos que hayan desarrollado malformaciones (Figura 3.A), sin embargo, en la muestra de Fray Bentos se encontró un $3.3 \%$ de malformaciones de la espina dorsal $(n=8)$, correspondientes a curvatura espinal en la región abdominal (Figura 3.B). Para el elutriado de Fray Bentos se aplicó el test de Kruskal Wallis (Steel y Torrie, 1993) con $\alpha=0.05$ en las concentraciones: control 100, 50, 25, 12.5 y 6.25 el test dio $\mathrm{KW}=11.6$ y $\mathrm{p}=0.04, \mathrm{p}<0.05$, lo cual constituye diferencias significativas. El número de malformaciones y mortalidad evidenciadas a la concentración más baja fueron mayores (35\%) que a las concentraciones más altas (Gráfico 1).

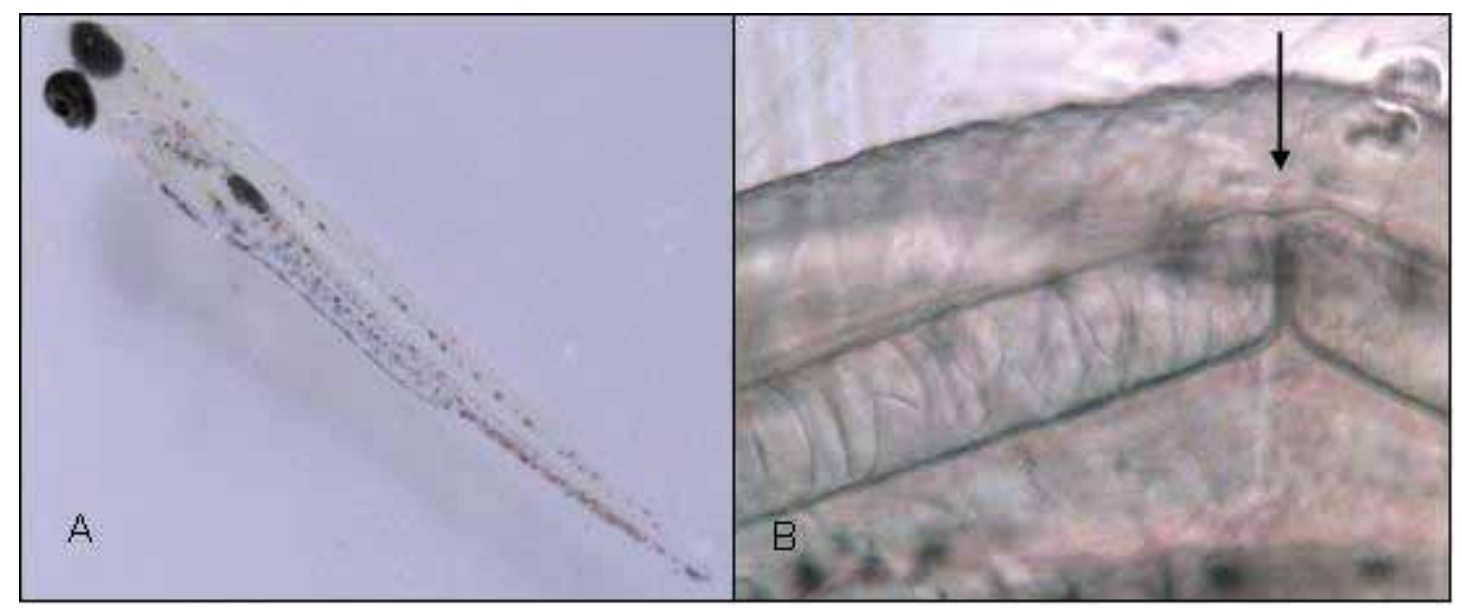

Figura 3. A. Larva de P. promelas de 6 días post fecundación (dpf) con desarrollo normal. B. Microfotografía (100x) de la espina dorsal de una Larva de $P$. promelas de elutriado de Fray Bentos de 6 dpf con anomalía de desarrollo en la curvatura de la espina. La flecha señala el punto donde ocurre discontinuidad espinal en la región abdominal.

Los ensayos preliminares para el sitio de Las Cañas no han demostrado ni mortalidad ni teratogenicidad. 


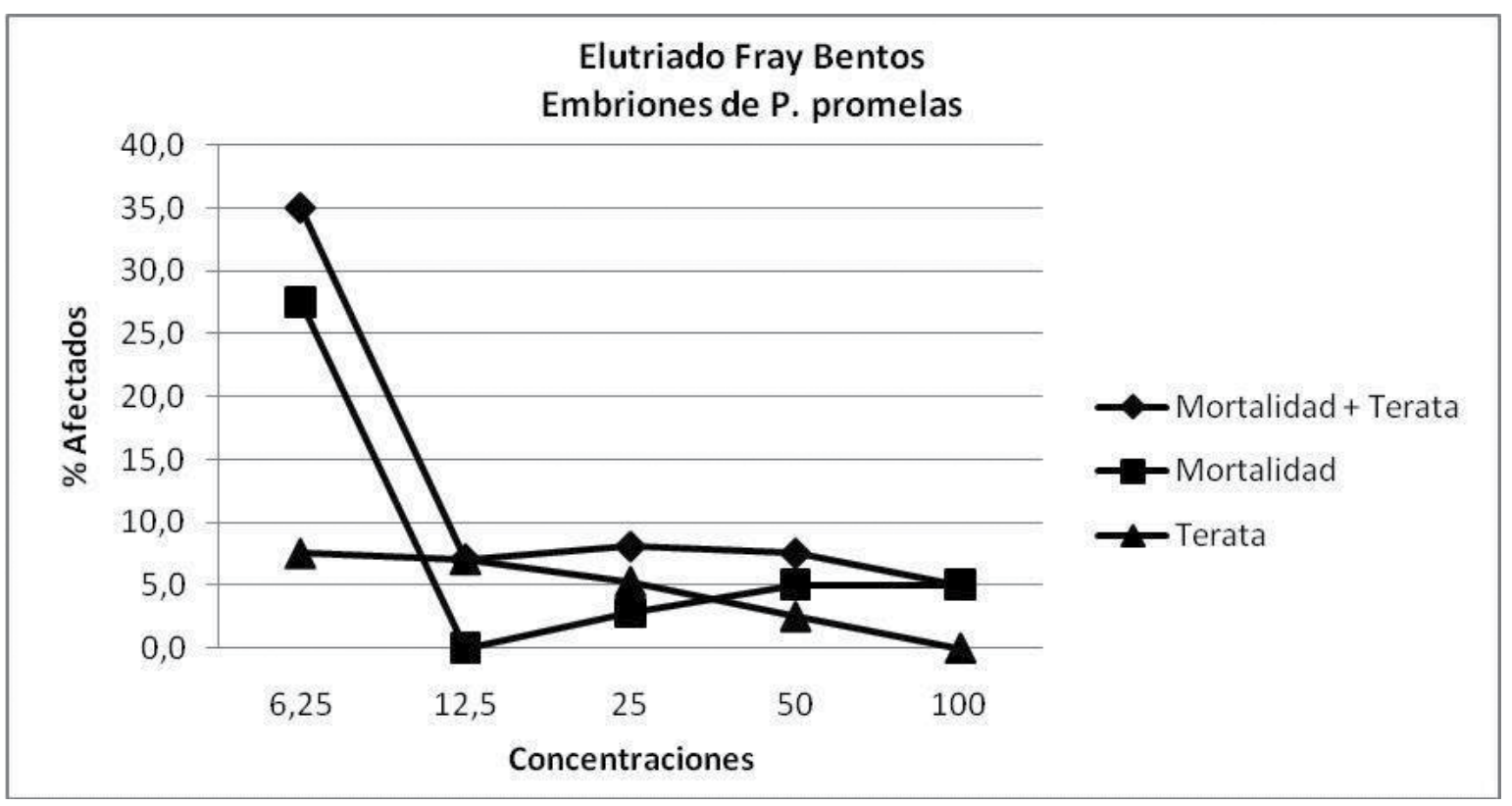

Gráfico 1. Curva dosis-respuesta para los efectos Mortalidad, Teratogenicidad y Mortalidad + Teratogenicidad de embriones de $P$. promelas en muestras de elutriado de sedimento del sitio Fray Bentos

\section{Discusión}

Los análisis químicos en sedimentos demuestran la influencia de las actividades antrópicas ya que el nivel de nutrientes de Fray Bentos fue mayor que en Nuevo Berlín. La proporción de arcilla de los sitios Fray Bentos y Nuevo Berlín es mucho mayor que en Las Cañas, posiblemente concentrando los contaminantes ambientales en esta fracción. El fraccionamiento de EOX y de AOX del compuesto clorado 2,4,5-TCP fue medido demostrando que éste se reparte entre las fases.

De acuerdo a algunos autores, el ensayo de screening in vitro con P. leighonati es muy sensible (Ulitzur et al., 2002; Broers y Lappalainen, 2004). Nuestros resultados experimentales permitieron detectar toxicidad por metales y compuestos orgánicos en todas las zonas, pero especialmente en el sitio de Fray Bentos.

Los resultados de los bioensayos con C. dubia mostraron efectos tóxicos sobre la reproducción en Nuevo Berlín y en Las Cañas.

El crecimiento de $H$. curvispina luego de la exposición a estos sedimentos fue significativamente menor que el crecimiento obtenido en el control. Se postula que dicha disminución podría deberse a la ingestión de partículas de sedimento con contaminantes asociados. Para este bioensayo se encontró que el crecimiento de los organismos fue significativamente menor luego de la exposición a las tres muestras analizadas (Nuevo Berlín, Fray Bentos y Las Cañas), respecto al control. Sin embargo, no se encontraron diferencias significativas en el crecimiento promedio entre las tres muestras analizadas, lo cual sugiere que un potencial efecto tóxico en estos organismos se podría estar dando por igual para los tres puntos de muestreo.

En el sitio de Fray Bentos fue observado un efecto estadísticamente de borde para el punto final de desarrollo en embriones de peces, consistente en deformaciones de la espina dorsal columna vertebral de frecuencia aún cercana a los valores considerados normales en $P$. promelas (Warner y Jenkins, 2007) y Cyprinus carpio (Lugowska, 2007). La curva dosis-respuesta observada para la aparición de las malformaciones vertebrales sigue un comportamiento no-monotónico.
Este hallazgo correlaciona con los encontrados previamente por otros investigadores para estos efectos (Warner y Jenkins, 2006). Aun cuando las malformaciones embrionales en la espina dorsal pudieron haberse producido por causas genéticas u otros factores ambientales, Villeneuve et al. (2005) encontró que valores mayores a $4 \%$ de malformaciones podrían ser indicativos de disrupción endócrina. La disrupción endócrina puede no limitarse a puntos de reproducción, ya que los receptores estrogénicos están presentes también en las células óseas. El estrógeno endógeno inicia la expresión de los elementos de respuesta estrogénica en osteoblastos y osteoclastos, regulando la proliferación de células óseas. Entonces, los disruptores estrógenos exógenos pueden interrumpir el control de la osificación (Warner y Jenkins, 2007). La presencia de larvas de peces $(n=8)$ con malformaciones de la curvatura dorsal correlaciona con los hallazgos de Weis y Weis (1987) quienes proponen que el desarrollo del sistema esquelético es uno de los más sensibles a sustancias teratógenas. Las potenciales consecuencias de los defectos espinales producirían una merma en las habilidades de presa, defensa y cortejo (Warner y Jenkins, 2007). Las malformaciones de este tipo en el orden Cipriniformes han sido demostradas con metales tales como cadmio y cadmio/cobre (Lugowska, 2007), y en P. promelas Laban et al. (2010) y Warner y Jenkins (2007) obtuvieron similares resultados después de exponer embriones y larvas a nanopartículas de plata y $17 \alpha$ etinilestradiol y Bisphenol A, respectivamente. Algunos investigadores han extendido el tiempo de exposición a más de 20 días usando P. promelas (Warner y Jenkins, 2007) y Cyprinus carpio (Lugowska, 2007); demostraron que algunas anomalías se podrían deber a exposición a esta clase de contaminantes. Sin embargo, Lugowska (2007) también encontró algunas malformaciones de este tipo que mejoran después de cultivar las larvas en condiciones óptimas. Los posibles contaminantes causantes de deformidades esqueléticas en peces son variados, incluyendo, entre otros, a metales pesados tales como plomo (Jezierska et al., 2009), pesticidas organofosforados, dioxinas y bifenilos policlorados (Villeneuve et al., 2005). 


\section{Conclusiones}

Los resultados preliminares sugieren que existen indicios de toxicidad y teratogenicidad de origen todavía no claramente establecido, pero que presentan diferente respuesta de toxicidad para los puntos finales incluidos en el experimento según el sitio. Los efectos evidenciados con organismos que se nutren del sedimento así como los de las especies que viven en el agua de columna apuntan a que las rutas de toxicidad en los sedimentos del Río Uruguay analizados no serían solamente a través de las sustancias contaminantes disueltas, sino también a través de las partículas en suspensión.

Este estudio proseguirá evaluando las diferencias de biodisponibilidad y toxicidad entre sitios y las diversas sensibilidades de los organismos ante posibles tóxicos ambientales. El compuesto 2,4,5-TCP se utilizará como modelo e incluirá métodos que miden biomarcadores de disrupción endocrina para proseguir generando evidencias sobre los probables contaminantes ambientales señalados en la identificación de peligros en virtud de las fuentes, receptores y rutas de contaminación más probables. En vista de estos datos preliminares, se continuarán las investigaciones para poder establecer con mayor certeza los vínculos causa-efecto.

\section{Reconocimientos}

El trabajo experimental se realizó en el Laboratorio Tecnológico del Uruguay (LATU).

Los autores agradecen a todo el personal del departamento Aguas y Productos Químicos por su apoyo a las actividades analíticas y al personal del departamento de Medio Ambiente por el muestreo de los sedimentos.

Los autores agradecen al Dr. James Lazorchak y su equipo de USEPA por proporcionar las cepas iniciales de los cultivos de C. dubia y P. promelas.

Este trabajo se desarrolló como parte de un proyecto de doctorado de investigación $\mathrm{PhD}$ de Cranfield University, School of Applied Sciences, Centre for Water Science, Cranfield, UK, apoyado por el Laboratorio Tecnológico del Uruguay.

\section{Referencias}

- ATSDR. Toxicological profile for chlorophenols [En línea]. Atlanta: ATSDR, s.d. [Consulta: 1 de julio de 2010]. Disponible en: http://www.atsdr.cdc.gov/ToxProfiles/tp107.pdf

- BROERS, Cam; LAPPALAINEN, J. New developments in the bioluminiscence assay [En línea]. En: Courier du Savoir. 2004, (5):107-110. [Consulta: 10 de junio de 2010]. Disponible en: http:// www.webreview.dz/IMG/pdf/16-Broers.pdf.

- BURT, Rebecca. Soil survey laboratory methods manual. v. 4.0. Lincoln: NRCS, 2004. (Soil Survey Investigations Reports: 42).

- CHAPMAN, Peter M. Integrating toxicology and ecology: putting the "eco" into ecotoxicology. En: Marine Pollution Bulletin. 2002,44:7-15

- DEWIS, J.; FREITAS, F. Métodos físicos y químicos de análisis de suelos y aguas. Roma: FAO, 1984. (Boletín de Suelos de la FAO $10)$.

- HALDER, M.; LEONARD, M.; IGUCHI, T.; ORIS, J.; RYDER, K.; BELANGER, S.; BRAUNBECK, T.; EMBRY, M.; WHALE, G.; NORBERG-KING, T. \& LILLICRAP, A. Regulatory aspects on the use of fish embryos in Environmental Toxicology. En: Integrated Environmental Assesment and Management. 2010, 6(3):484-491

- INTERNATIONAL STANDARD ORGANIZATION (Switzerland).
ISO 9562: Water quality. Determination of adsorbable organically bound halogens (AOX). Geneva: ISO, 2004.

- INTERNATIONAL STANDARD ORGANIZATION (Switzerland). ISO 5663: Water quality. Determination of Kjeldahl nitrogen. Method after mineralization with selenium. Geneva: ISO, 1984.

- JEZIERSKA, BARBARA; ŁUGOWSKA, KATARZYNA; WITESKA, MAŁGORZATA. The effects of heavy metals on embryonic development of fish (a review).En: Fish Physiology and Biochemistry. 2009, 35:625-640.

- KATAGI, T. Behavior of pesticides in water-sediment systems. En: Reviews of Environmental Contamination and Toxicology. 2006, 187:133-251.

- KENDAL, R.J. et al. Ecotoxicology. En: KLAASSEN, Curtis D.; CASARETT; DOULL'S. Toxicology: the basic science of poisons. v. 29. 6th ed. New York: McGraw-Hill, 2001.

- LABAN, G.; NIES, L.; TURCO, R.; BICKHAM, J.; SEPÚLVEDA, $M$. The effects of silver nanoparticles on fathead minnow (Pimephales promelas) embryos. En: Ecotoxicology. 2010, 19:185195.

- LUGOWSKA, K. The effect of cadmium and cadmium/copper mixture during the embryonic development on deformed common carp larvae [En línea]. En: Electronic Journal of Polish Agricultural Universities. 2007, 10(4). [Consulta 1 de julio de 2010]. Disponible en: http://www.ejpau.media.pl/volume10/issue4/art-11.html

- MARTÍN-DíAZ, M.L.; RIBA, I.; CASADO-MARTÍNEZ M.C.; DELLVALLS, A. Biodisponibilidad de metales en sedimentos de estuarios españoles utilizando Carcinus maeñas. En: Ciencias Marinas. 2006, 32(2B):412-420

- MIGUEZ, D.; MARTINEZ, M.J.; DOL, I. Desarrollo del método análisis por inyección en flujo para la determinación de fósforo total en sedimentos. En: 1er. Congreso Uruguayo de Química AnalíticaCUQA, (Montevideo 9-10 de setiembre de 2009). Montevideo: s.n., 2009.

- NATIONAL ACADEMY OF SCIENCES, NAS. [En línea]. En: Bioavailability of Contaminants in Soils and Sediments: Processes, Tools, and Applications. Committee on Bioavailability of Contaminants in Soils and Sediments, National Research Council. Disponible en: http://www.nap.edu/catalog/10523.html [Consulta: 26/10/2010].

- OHLENBUSCH, GERD; KUMKE MICHAEL, U; FRIMMEL, FRITZ H. Sorption of phenols to dissolved organic matter investigated by solid phase microextraction. En: The Science of the Total Environment. 2000, 253:63-74.

- SASKI, EIJA. K; MIKKOLA, R; KUKKONEN, JUSSI, V.K.; SALKINOJA-SALONEN, MIRJA S. Bleached Kraft pulp mill discharged organic matter in recipient lake sediment. En: Environmental Science and Pollution Research. 1997, 4(4):194-202.

- SIBLEY, P.K.; DIXON, D.G.; BARTON, D.R. Environmental assessment of benthic impacts associated with pulp mill discharges. II. Distribution of sediment EOX in relation to environmental factors. En: Archives of Environmental Contamination and Toxicology. 1998, 34:158-166

- STEEL, R.; TORRIE, J. Bioestadística. Principios y procedimientos. 2a. ed. México: McGraw-Hill, 1993, 651 pp.

- ULITZUR, S.; LAHAV, T.; ULITZUR, N. A novel and sensitive test for rapid determination of water toxicity. En: Environmental Toxicology. 2002, 17:291-296.

- UNESCO; WHO; UNEP. Water quality assessments: a guide to use of biota, sediments and water in environmental monitoring. 2nd. ed. London: University Press, 1996.

- UOP A HONEYWELL COMPANY. UOP methods. s.l.: UOP, 2007. 
UOP method 856-07.

- UNITED STATES OF AMERICA. UNITED STATES ENVIRONMENTAL PROTECTION AGENCY. [EPA's sampling and analysis methods]. [En linea]. Washington: USEPA, [s.d.]. Method 823-R-00-001, 2000. [Consulta 2 de julio de 2010]. Disponible en: http://www.epa.gov/waterscience/cs/biotesting/bioaccum.pdf

- UNITED STATES OF AMERICA. UNITED STATES ENVIRONMENTAL PROTECTION AGENCY. [EPA's sampling and analysis methods]. [En linea]. Washington: USEPA, [s.d.]. Method 9023, 1996. [Consulta 2 de julio de 2010]. Disponible en: http:// www.epa.gov/wastes/hazard/testmethods/sw846/pdfs/9023.pdf

- UNITED STATES OF AMERICA. UNITED STATES ENVIRONMENTAL PROTECTION AGENCY. [EPA's sampling and analysis methods]. [En linea]. Washington: USEPA, [s.d.]. Method 823-B-98-004,1998. [Consulta 2 de julio de 2010]. Disponible en: http://www.epa.gov/owow/oceans/regulatory/dumpdredged/pdf/ itm_feb1998.pdf

- UNITED STATES OF AMERICA. UNITED STATES ENVIRONMENTAL PROTECTION AGENCY. [EPA's sampling and analysis methods]. [En linea]. Washington: USEPA, [s.d.]. Method 821-R-02-013. [Consulta 2 de julio de 2010]. Disponible en: http:// www.epa.gov/

- VILLENEUVE, D.L.; CURTIS, L.R.; JENKINS, J.J.; WARNER, K.E.; TILTON, F.; KENT, M. L.; WATRAL, V.G.; CUNNINGHAM, M.E.; MARKLE, D.G.; SETAJINTANIN, D.; KRISSANAKRIANGKRAI, O.; JOHNSON, E.R.; GROVE, R.; ANDERSON, K.M. Environmental stresses and skeletal deformities in fish from the Willamette River. En: Environmental Science and Technology. 2005, 39(10):3495-3506.

- VOUTSAS, EPAMINONDAS; MAGOULAS, KOSTIS; TASSIOS, DIMITRIOS. Prediction of the bioaccumulation of persistent organic pollutants in aquatic food webs. En: Chemosphere. 2002, 48:645-651

- WARNER, K.; JENKINS, J. Effects of 17 $\alpha$-Ethinylestradiol and Bisphenol A on vertebral development in the fathead minnow (Pimephales promelas). En: Environmental Toxicology and Chemistry. 2007, 26(4):732-737.

- WEIS, J.; WEIS, P. Pollutants as developmental toxicants in aquatic organisms. En: Environmental Health Perspectives. 1987, 71:77-85. 